\title{
Quantitative Diffusion-Weighted MRI Parameters and Human Papillomavirus Status in Oropharyngeal Squamous Cell Carcinoma
}

\author{
C.S. Schouten, P. de Graaf, E. Bloemena, B.I. Witte, B.J.M. Braakhuis, R.H. Brakenhoff, C.R. Leemans, J.A. Castelijns, and R. de Bree
}

\begin{abstract}
BACKGROUND AND PURPOSE: Patients with human papillomavirus-positive oropharyngeal squamous cell carcinomas have a better survival rate than those with human papillomavirus-negative oropharyngeal squamous cell carcinomas. DWI characterizes biologically relevant tumor features, and the generated ADC may also provide prognostic information. We explored whether human papillomavirus status and ADC values are independent tumor characteristics.
\end{abstract}

MATERIALS AND METHODS: Forty-four patients with oropharyngeal squamous cell carcinomas underwent pretreatment DWI. ADC values for the primary tumors were determined by using 3 b-values in an ROI containing the largest area of solid tumor on a single section of an axial DWI image. Human papillomavirus status was determined with p16 immunostaining, followed by high-risk human papillomavirus DNA detection on the p16-positive cases.

RESULTS: Twenty-two patients were human papillomavirus-positive (50.0\%). ADC values were not significantly different between human papillomavirus-negative $\left(\mathrm{ADC}_{\text {mean }}=1.56[1.18-2.18] \times 10^{3} \mathrm{~mm}^{2} / \mathrm{s}\right)$ and human papillomavirus-positive tumors $\left(A D C_{\text {mean }}=\right.$ $\left.1.46[1.07-2.16] \times 10^{3} \mathrm{~mm}^{2} / \mathrm{s}\right)$.

CONCLUSIONS: No significant association between ADC and human papillomavirus status was found in oropharyngeal squamous cell carcinomas. In our study population, differences in genetic and histologic features between human papillomavirus-positive and human papillomavirus-negative oropharyngeal squamous cell carcinomas did not translate into different ADC values. Long-term follow-up studies are needed to establish whether $A D C$ has prognostic value and whether this is independent of the human papillomavirus status.

ABBREVIATIONS: HNSCC = head and neck squamous cell carcinoma; $\mathrm{HPV}=$ human papillomavirus; $\mathrm{OPSCC}=$ oropharyngeal squamous cell carcinoma

$\mathrm{H}^{\circ}$ ead and neck squamous cell carcinoma (HNSCC) is the sixth most common cancer worldwide. ${ }^{1}$ During the past decades, it has been well-established that besides tobacco smoking and alcohol consumption, human papillomavirus (HPV) is an important etiologic factor in the development of HNSCC, in particular squamous cell carcinomas in the oropharynx (OPSCC). HPVpositive and HPV-negative OPSCCs are different disease entities. ${ }^{2}$ Patients with HPV-positive OPSCC show higher response rates to treatment and have a better overall survival compared with those

Received September 16, 2014; accepted after revision October 8.

From the Departments of Otolaryngology-Head and Neck Surgery (C.S.S., B.J.M.B., R.H.B., C.R.L., R.d.B.), Radiology and Nuclear Medicine (P.d.G., J.A.C.), Pathology (E.B.), and Epidemiology and Biostatistics (B.I.W.), VU University Medical Center, Amsterdam, the Netherlands; and Department of Oral and Maxillofacial Surgery/Oral Pathology (E.B.), VU University Medical Center/Academic Center for Dentistry Amsterdam, Amsterdam, the Netherlands.

Paper previously presented at: Dutch National Otolaryngology Conference, April 24-25, 2014; Nieuwegein, the Netherlands.

Please address correspondence to R. de Bree, MD, Department of Otolaryngology-Head and Neck Surgery, VU University Medical Center, De Boelelaan 1117, 1081 HV Amsterdam, the Netherlands; e-mail: r.bree@vumc.nl

http://dx.doi.org/10.3174/ajnr.A4271 with HPV-negative OPSCC, despite the more often regionally advanced disease presentation in patients with HPV-positive OPSCC. ${ }^{3,4}$ In addition, the genetic route to cancer is different for HPV-positive OPSCC, ${ }^{5,6}$ and HPV-related tumors have distinct histologic features. ${ }^{7,8}$ In this context, traditional prognostic factors such as tumor size and lymph node invasion may be insufficient to fully classify patients into risk groups. Identification of other prognostic tumor characteristics may lead to an improved patient selection and, as a result, higher responses to treatment and probably less treatment-induced morbidity.

DWI is a noninvasive functional technique that characterizes tissue on the basis of the random motion of water molecules, which is mainly influenced by the volume of extracellular space and the presence of cell membranes. Differences in water mobility can be quantified with the ADC: Hypocellular or necrotic tissue or both are characterized by a high ADC, whereas hypercellular tissue is characterized by a low ADC. Hence, parameters from DWI, such as ADC, could indicate biologic dissimilarities among tumors. Furthermore, studies in breast cancer have demonstrated that the ADC value significantly correlates with the expression of specific bi- 
ologic markers of disease, such as estrogen receptor and progesterone receptor. ${ }^{9,10}$ ADC is a possible prognostic factor in HNSCC; several studies have shown that HNSCC with relatively low pretreatment ADC values responds better to chemoradiotherapy than tumors with higher pretreatment ADC values. ${ }^{11-14}$ Additionally, HPV-positive OPSCC often has a different histology from HPV-negative OPSCC, characterized by ovoid-to-spindle-shaped hyperchromatic cells without keratinization and without a stromal response, ${ }^{15}$ and it can by hypothesized that these histologic characteristics translate into low pretreatment $\mathrm{ADC}$ values; if so, this hypothesis would support the general notion that low ADC correlates with a better response to chemoradiotherapy. Limited information is available on the relation between HPV involvement and ADC values. ${ }^{16}$

In this study, we investigated the possible association between ADC values derived from DWI and the presence of biologically active HPV in patients with OPSCC.

\section{MATERIALS AND METHODS Patients and Study Design}

The study was approved by the institutional review board of VU University Medical Center. We identified patients with a histopathologically proved OPSCC who had undergone DWI for diagnosis and treatment planning between January 2010 and December 2013. Patients with T1 oropharyngeal tumors were excluded because a reliable ROI could not be drawn. Fifty-seven patients could be evaluated. Medical records were reviewed for clinical characteristics, including smoking and alcohol intake, tumor node metastasis stage, and oropharyngeal subsite. Three patients were excluded due to insufficient quality of the DWI. In 2 other patients, the primary tumor was outside the range of obtained MR diffusion images; therefore, they were excluded. Eight more patients were excluded because DWI was performed with only 2 b-values. Thus, 44 patients were enrolled in the DWI analysis.

\section{HPV Analysis}

HPV testing was performed on all tumors by using a previously defined and validated algorithm for HPV detection. ${ }^{17,18}$ In short, formalin-fixed and paraffin-embedded tumor tissue was stained by immunohistochemistry for p16 (the product of the cyclindependent kinase inhibitor 2A); and on the p16-immunopositive cases, high-risk HPV DNA was detected with general primer $5+/ 6+$ polymerase chain reaction. Only the cases that were positive in the latter assay were classified as HPV-positive.

\section{DWI}

All MR imaging examinations were performed by using a $1.5 \mathrm{~T}$ MR imaging system (Signa HDx; GE Healthcare, Milwaukee, Wisconsin) with a head coil combined with a phased array spine and neck coil. After an axial STIR series with 7-mm sections covering the entire neck area, subsequent images were centered on the area of interest containing the primary tumor and enlarged lymph nodes. Axial images (22 sections of 4-mm section thickness and 0.4-mm gap) were obtained with STIR (TR/TE/T1 = 6600/ $60 / 160 \mathrm{~ms}, 2$ averages, in-plane pixel size of $0.7 \times 1.1 \mathrm{~mm})$ and T1WI spin-echo $(\mathrm{TR} / \mathrm{TE}=500 / 14 \mathrm{~ms}, 2$ averages, no fat saturation, in-plane pixel size of $0.5 \times 0.5 \mathrm{~mm}$ ) before and after the intravenous injection of contrast material: Dotarem $(0.2 \mathrm{~mL} / \mathrm{kg}$ of gad- oteric acid; Guerbet, Aulnay-sous Bois, France) or Gadovist (0.1 $\mathrm{mL} / \mathrm{kg}$ of gadobutrol; Bayer Schering Pharma, Berlin, Germany).

DWI by using a PROPELLER technique was performed for 16 sections at the same section positions as the axial STIR and T1WI. Parameters for PROPELLER DWI were the following: TR/TE $=$ $3500 / 83.87 \mathrm{~ms}$; in-plane pixel size $=2 \times 2 \mathrm{~mm}$; and $\mathrm{b}$-values $=0$, 750 , and $1000 \mathrm{~s} / \mathrm{mm}^{2}$ (3 averages). ADC maps were calculated online or off-line, respectively, by using the software of the scanner.

DWI scans were analyzed with AEGIS (Aegis Web, Version 3.2.4; Hologic, Bedford, Massachusetts), which allowed viewing of multiple MR images. The primary tumors were first identified on conventional MR images. ADC values were measured by drawing an ROI on a single section of an axial high-b-value DWI containing the largest area of solid tumor, excluding any necrotic regions with the aid of postcontrast MR images. If artifacts within the lesion were present, a smaller ROI was placed only over the undistorted area of the lesion. Subsequently, the ROIs were copied to the corresponding $\mathrm{ADC}$ maps. $\mathrm{ADC}$ was obtained as $\mathrm{ADC}_{\text {minimum }}$ (lowest tumor voxel value within the $\mathrm{ROI}$ ) and $\mathrm{ADC}_{\text {mean }}$ (mean $\mathrm{ADC}$ within the ROI). The ROIs were outlined by C.S.S. (MD, PhD candidate) and confirmed by a board-certified head and neck radiologist with 5 years of experience in head and neck radiology (P.d.G). Clinical information about the tumor node metastasis stage was known, but the interpreters were blinded to HPV status.

\section{Statistical Analysis}

Statistical analyses were performed by using the SPSS software package (Version 20.0; IBM, Armonk, New York). The level of significance was set at $P<.05$, and hypotheses were tested 2 -sided. Analyses for differences in patient characteristics between the HPV-positive and HPV-negative groups were performed with the Pearson $\chi^{2}$ test for categoric data and the Fisher exact test when appropriate. A Bonferroni correction was used to compare subgroups. The Mann-Whitney $U$ test was used to compare continuous data.

\section{RESULTS}

The total study group consisted of 33 men and 11 women, with a mean age at the time of diagnosis of 58.8 years (range, 45-78). Patient characteristics by HPV status are shown in Table 1. Patients who were HPV-positive were more likely to present with a T2 primary tumor and N-positive disease. Patients who were HPV-negative were more likely to have a history of heavy smoking and excessive alcohol consumption.

With DWI, ROI areas for OPSCC ranged from 20.0 to 770.0 $\mathrm{mm}^{2}$. The median ROI area for HPV-positive and HPV-negative OPSCCs was $165.0 \mathrm{~mm}^{2}$ (range, $40-450 \mathrm{~mm}^{2}$ ) and $160.0 \mathrm{~mm}^{2}$ (range, $20-770 \mathrm{~mm}^{2}$ ), respectively. The ROI area did not significantly differ between patients who were HPV-positive and those who were HPV-negative $(P=.77)$. Examples of a DWI scan from a patient who was HPV-negative and one who was HPV-positive are shown in Figs 1 and 2, respectively. The results did not demonstrate significant differences between $\mathrm{ADC}_{\text {mean }}$ and $\mathrm{ADC}_{\text {minimum }}$ in the HPV-negative and HPV-positive groups: $\mathrm{ADC}_{\text {mean }}$ and $\mathrm{ADC}_{\text {minimum }}$ were $1.46(1.07-2.16) \times 10^{-3} \mathrm{~mm}^{2} / \mathrm{s}$ (median [range] ) and $1.01(0.62-1.55) \times 10^{-3} \mathrm{~mm}^{2} / \mathrm{s}$ for patients who were HPV-positive and $1.56(1.18-2.18) \times 10^{-3} \mathrm{~mm}^{2} / \mathrm{s}$ and $1.07(0.62-1.51) \times 10^{-3} \mathrm{~mm}^{2} / \mathrm{s}$ for patients who were HPV-negative ( $P=.51$ and $P=.67$, respectively) (Table 2$)$. 
Table 1: General patient characteristics

\begin{tabular}{|c|c|c|c|}
\hline Characteristic & $\begin{array}{l}\text { HPV-Positive } \\
(n=22)(50 \%)\end{array}$ & $\begin{array}{l}\text { HPV-Negative } \\
(n=22)(50 \%)\end{array}$ & $\begin{array}{c}P \\
\text { Value }\end{array}$ \\
\hline Sex & & & .30 \\
\hline Male & $18(81.8 \%)$ & $15(68.2 \%)$ & \\
\hline Female & 4 (18.2\%) & $7(31.8 \%)$ & \\
\hline Age at diagnosis (yr) & & & .92 \\
\hline Median (range) & $57(47-78)$ & $60.5(45-71)$ & \\
\hline Oropharyngeal subsite & & & .29 \\
\hline Tonsil & $12(54.4 \%)$ & $10(45.5 \%)$ & \\
\hline Base of tongue & $8(36.4 \%)$ & $6(27.3 \%)$ & \\
\hline Oropharynx nos & $2(9.1 \%)$ & $6(27.3 \%)$ & \\
\hline Smoking ${ }^{a}$ & & & .01 \\
\hline Never (0-5 pack years) & $8(36.4 \%)$ & $0(0 \%)$ & \\
\hline Moderate (6-24 pack years) & $5(22.7 \%)$ & $5(22.7 \%)$ & \\
\hline Heavy ( $>24$ pack years) & $9(40.9 \%)$ & 17 (77.3\%) & \\
\hline Alcohol consumption ${ }^{\mathrm{b}}$ & & & .02 \\
\hline Never (0) & $3(13.6 \%)$ & $0(0 \%)$ & \\
\hline Moderate (1-149 U years) & 15 (68.2\%) & $10(45.5 \%)$ & \\
\hline Heavy (>149 U years) & $4(18.2 \%)$ & $12(54.5 \%)$ & \\
\hline T-stage & & & .03 \\
\hline $\mathrm{T} 2$ & $12(54.5 \%)$ & $5(22.7 \%)$ & \\
\hline T3-4 & $10(45.5 \%)$ & $17(77.3 \%)$ & \\
\hline $\mathrm{N}$-stage & & & .04 \\
\hline No & $0(0 \%)$ & $5(22.7 \%)$ & \\
\hline $\mathrm{N} 1-3$ & $22(100 \%)$ & 17 (77.3\%) & \\
\hline M-stage & & & NA \\
\hline MO & $22(100 \%)$ & $22(100 \%)$ & \\
\hline M1 & $0(0 \%)$ & $0(0 \%)$ & \\
\hline
\end{tabular}

Note:-NA indicates not applicable; Oropharynx nos, oropharynx not otherwise specified.

${ }^{a}$ Smoking was defined in pack years (1 pack year $=20$ cigarettes a day during 1 year). ${ }^{\mathrm{b}}$ Alcohol consumption was defined in unit years $(1 \mathrm{U}$ year $=1$ alcohol-containing consumption a day during 1 year).

\section{DISCUSSION}

OPSCCs may have a heterogeneous response to treatment, and survival varies among different groups of patients. ${ }^{3,4,19}$ Tobacco smoking, alcohol consumption, and HPV play a role in this heterogeneity, and in particular, an HPV infection is a strong independent prognostic factor for survival in OPSCC. ${ }^{4}$ Because traditional prognostic factors such as tumor size and lymph node involvement may be inadequate to classify patients into risk groups, identification of new prognostic tumor characteristics that may select patients for more or less aggressive treatment regimens becomes of interest. DWI may have prognostic value in HNSCC. ${ }^{11-14}$ It is not known, however, whether the relatively low pretreatment ADC values, which are associated with better response to chemoradiotherapy in these studies, could be attributed to HPV status. This study shows that imaging parameters derived from DWI are not significantly associated with the OPSCC HPV status. Therefore, HPV status and ADC values may be factors that independently determine the prognosis of a patient with OPSCC.

HPV-related OPSCC is a clinically and histopathologically distinct disease entity. ${ }^{20,21}$ In this study, we also found significant differences in clinical presentation, depending on HPV status; HPV-patients were more likely to have a T2 primary tumor but positive N-stage. Histopathologically, HPV-related tumors often have distinct features, characterized by a nonkeratinizing morphology and showing excessive mitoses and comedo-type necrosis, while HPV-negative OPSCC shows large polygonal cells and typical keratin formation. ${ }^{8,15,20-22}$ In general, HPV-positive OPSCC is more poorly differentiated. ${ }^{23}$ Characteristics of poorly differentiated squamous cell carcinomas, such as a higher cell attenuation
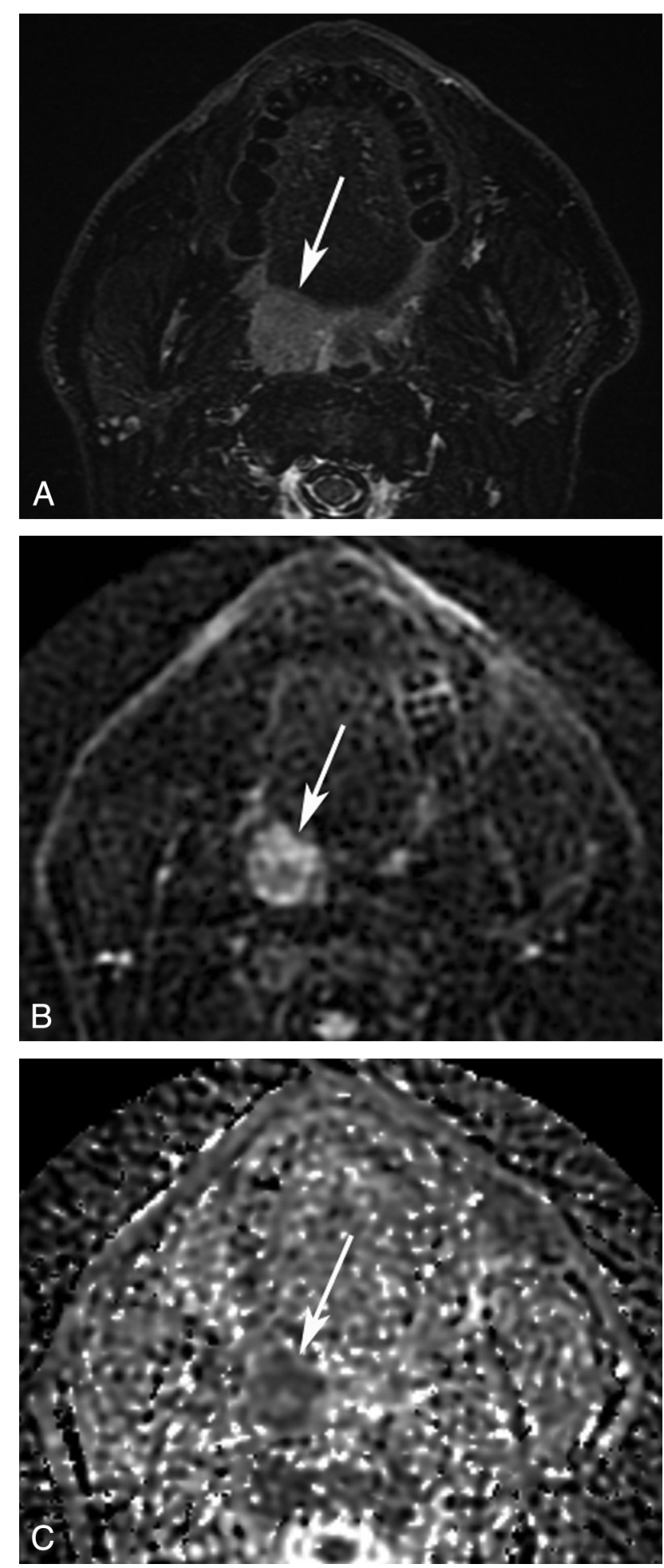

FIG 1. Axial MR images of an HPV-positive OPSCC. A 48-year-old patient was diagnosed with an OPSCC in the right palatine tonsil. $A$, STIR MR image shows a hyperintense mass in the palatine tonsil (arrow). A $b=750$ diffusion-weighted image $(B)$ and an ADC map (C) show decreased ADC. An ROI was drawn on the $b=750$ diffusionweighted image and copied to the ADC map. In this ROI, ADC $C_{\text {minimum }}$ measured $0.992 \times 10^{-3} \mathrm{~mm}^{2} / \mathrm{s}$.

and an increased nuclear-to-cytoplasmic ratio, reduce extracellular space and thereby the diffusion space of water protons, which could result in relatively low ADC values. ${ }^{24,25}$ Indeed, Wang et $\mathrm{al}^{24}$ found lower ADC values in poorly differentiated carcinomas

AJNR Am J Neuroradiol 36:763-67 Apr 2015 www.ajnr.org 

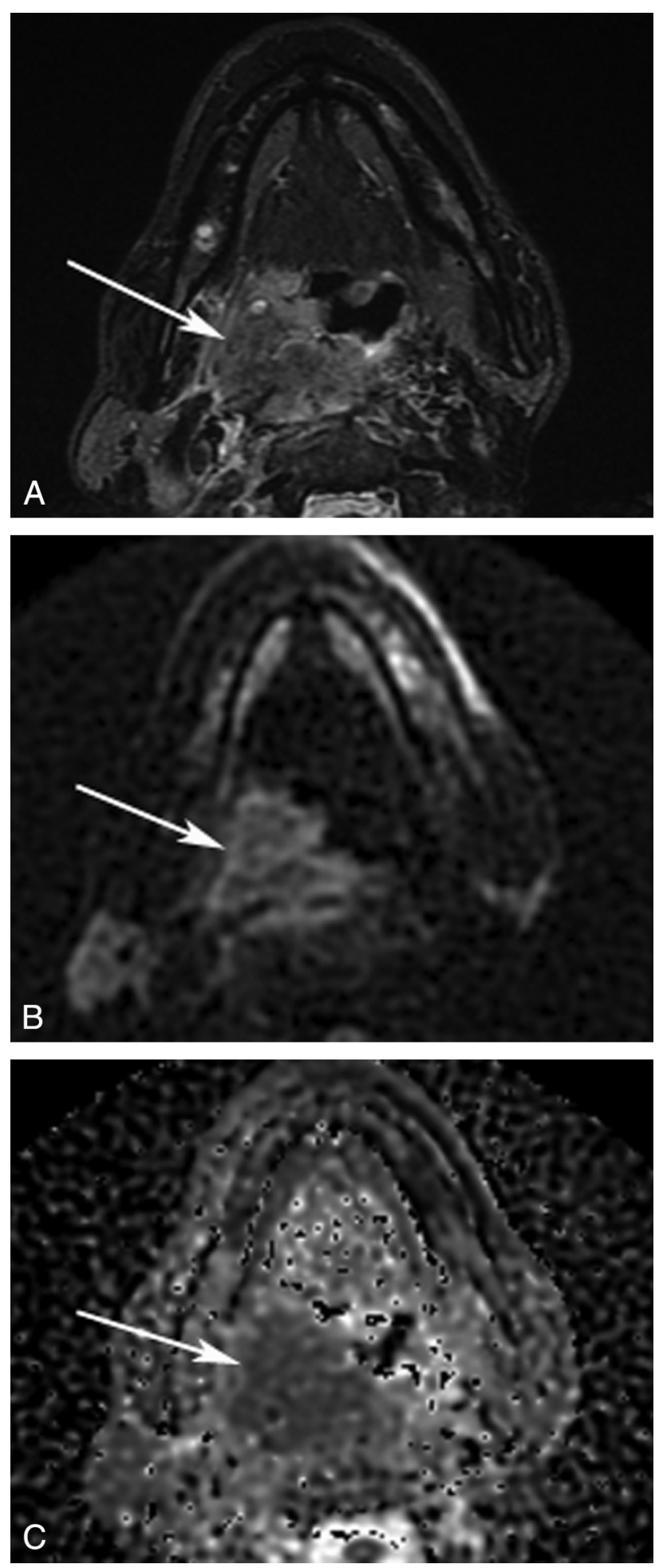

FIG 2. Axial MR images of an HPV-negative OPSCC. A 54-year-old patient was diagnosed with an OPSCC in the right palatine tonsil. $A$, STIR MR image shows a large hyperintense mass in the oropharynx (arrow). A $b=750$ diffusion-weighted image (B) and an ADC map (C) show decreased ADC. An ROI was drawn on the $b=750$ diffusionweighted image and copied to the ADC map. In this ROI, ADC minimum measured $0.826 \times 10^{-3} \mathrm{~mm}^{2} / \mathrm{s}$.

compared with well- or moderately differentiated carcinomas. It can be hypothesized that the distinct histologic features of HPVpositive and HPV-negative OPSCC may translate in different pretreatment $\mathrm{ADC}$ values. If this is the case, it would support the
Table 2: Imaging DWI parameters in relation to HPV status ${ }^{\mathrm{a}}$

\begin{tabular}{|c|c|c|c|c|c|}
\hline & No. & $\begin{array}{c}\mathrm{ADC}_{\min } \\
\left(\times 10^{3} \mathrm{~mm}^{2} / \mathrm{s}\right)\end{array}$ & $P$ Value & $\begin{array}{c}\mathrm{ADC}_{\text {mean }} \\
\left(\times 10^{3} \mathrm{~mm}^{2} / \mathrm{s}\right)\end{array}$ & $P$ Value \\
\hline HPV & & & .67 & & .51 \\
\hline Positive & 22 & $1.01(0.62-1.55)$ & & $1.46(1.07-2.16)$ & \\
\hline Negative & 22 & $1.07(0.62-1.51)$ & & $1.56(1.18-2.18)$ & \\
\hline
\end{tabular}

Note:-min indicates minimum.

${ }^{a}$ Values are presented as median (range).

general opinion that low ADC values correlate with a better response to chemoradiotherapy. ${ }^{11-14}$ Apparently, viable cells in a highly proliferating tumor (with low pretreatment ADC values) respond more favorably to chemoradiotherapy, probably related to the better vascularization that increases the exposure to chemotherapeutic agents. ${ }^{12,26}$ This response is in contrast to tissues with high levels of necrosis (with high ADC values), leading to a detrimental effect on treatment efficacy. ${ }^{27}$

In previous studies, ${ }^{1-14}$ an association was reported between treatment response and pretreatment ADC values. This reported association could partly or even completely be attributed to the HPV status because patients with an HPV-positive OPSCC respond better to chemoradiotherapy than those who are HPVnegative. Unfortunately, the HPV status of the OPSCC studied was not included in the analyses. As outlined before, HPV-positive OPSCC has a different histology, and HPV involvement could translate into different $\mathrm{ADC}$ values. We found no association between pretreatment $\mathrm{ADC}$ and HPV status, and this finding suggests that ADC value and HPV status are independent characteristics in patients with OPSCC, each with their own prognostic value.

Currently, limited data are available regarding the association between HPV status and ADC in patients with OPSCC. Our data are in contrast to those of Nakahira et al, ${ }^{16}$ who found significant lower pretreatment mean and minimum ADC values for HPVpositive than for HPV-negative OPSCCs. In their series of 26 patients, they used p16 protein overexpression as a surrogate marker for HPV infection. However, p16 immunohistochemistry is not an ideal surrogate marker for an HPV infection because a considerable percentage of patients are p16-positive but HPV-negative. ${ }^{17,28}$ The application of $\mathrm{p} 16$ protein overexpression as a surrogate marker for HPV infection could have affected their results. In our institution, we use a validated algorithm for HPV detection: p16 immunostaining, followed by a general primer $5+/ 6$ polymerase chain reaction on the p16-immunopositive cases. This algorithm showed an accuracy of $98 \% .{ }^{18}$ In addition, the conflicting data may also be explained by DWI factors; the choice of DWI-technique, the use of different b-values, and pulse sequences.

We acknowledge several limitations to this study. First, due to the retrospective study design that only included patients with an MR imaging with PROPELLER DWI, selection bias may have occurred. Second, the number of patients included was relatively small, and the study was performed in a single center. Finally, DWI studies were performed with a PROPELLER technique. DWI studies in HNSCC are most commonly performed with an echo-planar imaging sequence. ${ }^{11,29,30}$ DWI of the head and neck area is particularly difficult, because this region is very inhomogeneous and susceptible to artifacts. If artifacts are too detrimental, a non-EPI technique, such as PROPELLER, may be a better alternative. Nevertheless, in some primary lesions, artifacts were present. Consequently, a smaller ROI was placed over only the undistorted area of the lesion. 


\section{CONCLUSIONS}

Patients with HPV-positive OPSCC have a better survival rate compared with HPV-negative OPSCC. ADC values of DWI may also have prognostic value. In this study, we investigated a possible association between HPV status and ADC values in OPSCC. No significant associations were found between quantitative imaging parameters from DWI and HPV status in OPSCC. The differences in histologic features between HPV-positive and HPVnegative OPSCC did not translate into different pretreatment ADC values in our study cohort. Long-term follow-up studies are needed to investigate whether ADC values and HPV status are independent prognostic factors in patients with OPSCC treated by chemoradiotherapy.

\section{ACKNOWLEDGMENTS}

The authors thank M.M. Rietbergen and S. Hakim (Department of Otolaryngology-Head and Neck Surgery, VU University Medical Center, Amsterdam) for their assistance in retrieving HPV statuses and clinical characteristics. We also thank Daniel P. Noij (Department of Radiology and Nuclear Medicine, VU University Medical Center, Amsterdam) for assisting with ADC measurements.

Disclosures: Ruud Brakenhoff-UNRELATED: Grants/Grants Pending: European Union grants*; Patents (planned, pending or issued): tumor-specific lethal microRNAs. * Money paid to the institution.

\section{REFERENCES}

1. Ferlay J, Shin HR, Bray F, et al. Estimates of worldwide burden of cancer in 2008: GLOBOCAN 2008. Int J Cancer 2010;127:2893-917

2. Leemans CR, Braakhuis BJ, Brakenhoff RH. The molecular biology of head and neck cancer. Nat Rev Cancer 2011;11:9-22

3. Fakhry C, Westra WH, Li S, et al. Improved survival of patients with human papillomavirus-positive head and neck squamous cell carcinoma in a prospective clinical trial. J Natl Cancer Inst 2008;100:261-69

4. Ang KK, Harris J, Wheeler R, et al. Human papillomavirus and survival of patients with oropharyngeal cancer. $N$ Engl $\mathrm{J}$ Med 2010;363:24-35

5. Braakhuis BJ, Snijders PJ, Keune WJ, et al. Genetic patterns in head and neck cancers that contain or lack transcriptionally active human papillomavirus. J Natl Cancer Inst 2004;96:998-1006

6. Smeets SJ, Brakenhoff RH, Ylstra B, et al. Genetic classification of oral and oropharyngeal carcinomas identifies subgroups with a different prognosis. Cell Oncol 2009;31:291-300

7. Thariat J, Badoual C, Faure C, et al. Basaloid squamous cell carcinoma of the head and neck: role of HPV and implication in treatment and prognosis. J Clin Pathol 2010;63:857-66

8. Chernock RD, El-Mofty SK, Thorstad WL, et al. HPV-related nonkeratinizing squamous cell carcinoma of the oropharynx: utility of microscopic features in predicting patient outcome. Head Neck Pathol 2009;3:186-94

9. Martincich L, Deantoni V, Bertotto I, et al. Correlations between diffusion-weighted imaging and breast cancer biomarkers. Eur Radiol 2012;22:1519-28

10. Choi SY, Chang YW, Park HJ, et al. Correlation of the apparent diffusion coefficiency values on diffusion-weighted imaging with prognostic factors for breast cancer. Br J Radiol 2012;85:e474-79

11. Kim S, Loevner L, Quon H, et al. Diffusion-weighted magnetic resonance imaging for predicting and detecting early response to chemoradiation therapy of squamous cell carcinomas of the head and neck. Clin Cancer Res 2009;15:986-94

12. Srinivasan A, Chenevert TL, Dwamena BA, et al. Utility of pretreatment mean apparent diffusion coefficient and apparent diffusion coefficient histograms in prediction of outcome to chemoradiation in head and neck squamous cell carcinoma. J Comput Assist Tomogr 2012;36:131-37

13. Chawla S, Kim S, Dougherty L, et al. Pretreatment diffusionweighted and dynamic contrast-enhanced MRI for prediction of local treatment response in squamous cell carcinomas of the head and neck. AJR Am J Roentgenol 2013;200:35-43

14. Hatakenaka M, Nakamura K, Yabuuchi H, et al. Pretreatment apparent diffusion coefficient of the primary lesion correlates with local failure in head-and-neck cancer treated with chemoradiotherapy or radiotherapy. Int J Radiat Oncol Biol Phys 2011;81:339-45

15. Chernock RD, Lewis JS Jr, Zhang Q, et al. Human papillomavirus-positive basaloid squamous cell carcinomas of the upper aerodigestive tract: a distinct clinicopathologic and molecular subtype of basaloid squamous cell carcinoma. Hum Pathol 2010;41:1016-23

16. Nakahira M, Saito N, Yamaguchi H, et al. Use of quantitative diffusionweighted magnetic resonance imaging to predict human papilloma virus status in patients with oropharyngeal squamous cell carcinoma. Eur Arch Otorhinolaryngol 2014;271:1219-25

17. Smeets SJ, Hesselink AT, Speel EJ, et al. A novel algorithm for reliable detection of human papillomavirus in paraffin embedded head and neck cancer specimen. Int J Cancer 2007;121:2465-72

18. Rietbergen MM, Leemans $\mathrm{CR}$, Bloemena $\mathrm{E}$, et al. Increasing prevalence rates of HPV attributable oropharyngeal squamous cell carcinomas in the Netherlands as assessed by a validated test algorithm. Int J Cancer 2013;132:1565-71

19. Lassen P, Eriksen JG, Hamilton-Dutoit S, et al. Effect of HPV-associated p16INK4A expression on response to radiotherapy and survival in squamous cell carcinoma of the head and neck. J Clin Oncol 2009;27:1992-98

20. Gillison ML, Koch WM, Capone RB, et al. Evidence for a causal association between human papillomavirus and a subset of head and neck cancers. J Natl Cancer Inst 2000;92:709-20

21. El-Mofty SK, Patil S. Human papillomavirus (HPV)-related oropharyngeal nonkeratinizing squamous cell carcinoma: characterization of a distinct phenotype. Oral Surg Oral Med Oral Pathol Oral Radiol Endod 2006;101:339-45

22. El-Mofty SK, Zhang MQ, Davila RM. Histologic identification of human papillomavirus (HPV)-related squamous cell carcinoma in cervical lymph nodes: a reliable predictor of the site of an occult head and neck primary carcinoma. Head Neck Pathol 2008;2:163-68

23. Mendelsohn AH, Lai CK, Shintaku IP, et al. Histopathologic findings of HPV and p16 positive HNSCC. Laryngoscope 2010;120:1788-94

24. Wang J, Takashima S, Takayama F, et al. Head and neck lesions: characterization with diffusion-weighted echo-planar MR imaging. Radiology 2001;220:621-30

25. Sumi M, Sakihama N, Sumi T, et al. Discrimination of metastatic cervical lymph nodes with diffusion-weighted MR imaging in patients with head and neck cancer. AJNR Am J Neuroradiol 2003;24:1627-34

26. Kim S, Loevner LA, Quon H, et al. Prediction of response to chemoradiation therapy in squamous cell carcinomas of the head and neck using dynamic contrast-enhanced MR imaging. AJNR Am J Neuroradiol 2010;31:262-68

27. Tatum JL, Kelloff GJ, Gillies RJ, et al. Hypoxia: importance in tumor biology, noninvasive measurement by imaging, and value of its measurement in the management of cancer therapy. Int J Radiat Biol 2006;82:699-757

28. Rietbergen MM, Snijders PJ, Beekzada D, et al. Molecular characterization of p16-immunopositive but HPV DNA-negative oropharyngeal carcinomas. Int J Cancer 2014;134:2366-72

29. Vandecaveye V, Dirix P, De Keyzer F, et al. Predictive value of diffusion-weighted magnetic resonance imaging during chemoradiotherapy for head and neck squamous cell carcinoma. Eur Radiol 2010;20:1703-14

30. King AD, Mo FK, Yu KH, et al. Squamous cell carcinoma of the head and neck: diffusion-weighted MR imaging for prediction and monitoring of treatment response. Eur Radiol 2010;20:2213-20 